\title{
The effect of unilateral posteroventral pallidotomy on the kinematics of the reach to grasp movement
}

Kerry M B Bennett, John D O’Sullivan, Richard F Peppard, Peter M McNeill, Umberto Castiello

\begin{abstract}
Objective-to assess postoperative effects of unilateral posteroventral pallidotomy on the organisation of upper limb movement.

Methods-A three dimensional kinematic system (ELITE, B|T|S| Italy) was used to record reach to grasp movements to objects of either small $(0.7 \mathrm{~cm})$ or large $(8$ cm) diameter placed at a reaching distance of either 20 or $30 \mathrm{~cm}$. Four patients with Parkinson's disease were assessed in "off" (12 hours without medication) and "on" (1 hour after administration of medication) preoperatively and postoperatively.

Results-Duration of the movement and the time spent in arm deceleration were significantly reduced after surgery. However, movement patterning according to object size was adversely affected. Postoperatively, all four patients showed an abnormal pattern of a longer movement duration, and three showed a longer time of reaching arm deceleration, for reach to grasp movements to the large object than for those to the small object.

Conclusion-Posteroventral pallidotomy seems to be beneficial in reducing bradykinesia of upper limb movements but may have "costs" to movement patterning, particularly for reach to grasp movements to objects of differing sizes. This study raises interesting questions about the role of the globus pallidus interna in coordinating stimulus bound visual information with appropriate motor patterning.

(f Neurol Neurosurg Psychiatry 1998;65:479-487)
\end{abstract}

Keywords: Parkinson's disease; pallidotomy; kinematics; reach; grasp; human; movement

Clinical Neurosciences, St Vincent's Hospital, Melbourne, Australia R Peppard

P McNeill

Department of Psychology, University of Melbourne,

Australia

U Castiello

Correspondence to: Dr Kerry Bennett, Faculty of Health Sciences, La Trobe

University, Bundoora,

Victoria, Australia 3053

Telephone 006139479

5785 .

Received 4 June 1997 and in revised form 7 January 1998 Accepted 12 March 1998 the CAPIT score and of $68 \%$ on the UPDR score up to 12 months after pallidotomy. Similarly, Baron et $a l^{10}$ showed an average improvement of $30.1 \%$ on the UPDRS after 1 year. Although many studies report a lessening of bradykinesia and improvements to gait, ${ }^{124910}$ a recent study by Johansson et $a l^{6}$ found no changes to gait patterns or bradykinesia, but significant effects on tremor and dyskinesia induced by medication.

Despite calls from several researchers, ${ }^{12-15}$ very few studies have assessed the effectiveness of pallidotomy using objective measurement instruments. Bennett $e t a l^{16}$ reported the use of a three dimensional kinematic system to assess the reach to grasp movement of patients with Parkinson's disease preoperatively and postoperatively in both "off" (12 hours without medication) and "on" ( 1 hour after administration of medication) states. Preliminary results indicated that the movement was faster (by more than $150 \mathrm{~ms}$ ) after unilateral posteroventral pallidotomy. Using a computerised target location paradigm, Simuni et $a l^{17}$ also reported bilateral improvements postoperatively to movement speed, and in movement accuracy (see also Fookson et $a l^{18}$ ). Johansson et $a l^{6}$ conducted an optoelectronic analysis of a movement whereby medicated patients lifted an object from the floor and placed it on a shelf. Posteroventral pallidotomy resulted in no reduction in the time taken to perform this gross multicomponent action; however, more actions were performed per 30 second test sequence postoperatively, suggesting some shortening of the time between each action.

In the current study, a three dimensional kinematic system (ELITE, B|TISI Italy) was used to assess the effectiveness of surgery on an everyday action. This system allows for accurate measurement in both the temporal (accuracy $<10 \mathrm{~ms}$ ) and spatial (accuracy $<0.5 \mathrm{~mm}$ ) domains. It gives data about movement organisation, including its displacement, velocity and acceleration/deceleration profiles, in three dimensional space. It is thus a valuable tool for providing quantitative accurate measures of the effects of posteroventral pallidotomy on movement organisation.

The investigated movement was that of reaching to grasp an object, a natural everyday movement for which well used neuronal channels are recruited. As such, the learning or subject anxiety effects which may confound more experimental tasks are minimised. The reach to grasp movement has been well characterised by a series of studies beginning with the research of Jeannerod in the early 1980 s. ${ }^{19}$ It is said to 
Table 1 Scores for each section of the unified Parkinson's disease rating scale (UPDRS) preoperatively after withdrawal of medication for at least 12 hours (off state)

\begin{tabular}{lrrrr}
\hline \multirow{2}{*}{ Section of UPDRS } & \multicolumn{2}{l}{ Patient } & & \\
\cline { 2 - 5 } & \multicolumn{1}{l}{1} & 2 & \multicolumn{1}{l}{3} & 4 \\
\hline I Mentation, behavior, and mood & 1 & 4 & 3 & No data \\
II Activities of daily living & 21 & 13 & 18 & 16 \\
III Motor examination & 35 & 39 & 26 & 42 \\
IV Complications of treatment & 11 & 4 & 6 & 7 \\
Total & 68 & 60 & 53 & $65 / 131$ \\
\hline
\end{tabular}

consist of two principal components: (1) transport (or reach) which brings the hand to the appropriate spatial location, and (2) manipulation (or hand opening/closing) in which the hand opens to a peak and then closes appropriately on the target object.

Patients with Parkinson's disease in early disease stages and "on" medication generally show normal kinematic patterning of movement. Hence, when reaching to grasp an object, apart from the expected generalised slowing, patients with Parkinson's disease show the characteristic pattern of a longer deceleration phase of arm reaching and an earlier timing of maximum grip aperture for small than for large diameter objects. ${ }^{20-25}$ When reaching to grasp objects placed at different distances patients with Parkinson's disease show the characteristic pattern of an increase in the amplitude of peak reaching velocity and a relatively later hand opening peak for longer reaches. ${ }^{2023-26}$

Table 2 The results of a sample of clinical tests conducted before and after unilateral pallidotomy

\begin{tabular}{|c|c|c|c|c|}
\hline & \multicolumn{2}{|c|}{ Before } & \multicolumn{2}{|l|}{ After } \\
\hline & Off & On & Off & $\mathrm{On}$ \\
\hline \multicolumn{5}{|l|}{ Patient 1 (right pallidotomy): } \\
\hline Motor UPDR & 35 & 25 & 20 & 13 \\
\hline Hoehn and Yahr score & 3 & 3 & 2.5 & 2 \\
\hline Stand/walk 7m/sit (s) & 25 & 22.9 & 24.6 & 18.8 \\
\hline \multicolumn{5}{|l|}{ Two point test: } \\
\hline Right & 17.9 & 18.3 & 18.7 & 17.1 \\
\hline Left & 21.6 & 18.6 & 19.6 & 18.8 \\
\hline Total dyskinesia score & 0 & 10 & 0 & 8 \\
\hline Contralateral dyskinesia score & 0 & 4 & 0 & 1 \\
\hline \multicolumn{5}{|l|}{ Patient 2 (left pallidotomy): } \\
\hline Motor UPDR & 39 & 27 & 25 & 20 \\
\hline Hoehn and Yahr score & 2 & 2 & 2 & 2 \\
\hline Stand/walk $7 \mathrm{~m} / \mathrm{sit}(\mathrm{s}$ ) & 13.7 & 12.6 & 12.6 & 12.3 \\
\hline \multicolumn{5}{|l|}{ Two point test: } \\
\hline Right & 23.4 & 20 & 12.1 & 10.4 \\
\hline Left & 21.7 & 19 & 14.7 & 11.9 \\
\hline Total dyskinesia score & 0 & 0 & 0 & 0 \\
\hline \multicolumn{5}{|l|}{ Patient 4 (left pallidotomy): } \\
\hline Motor UPDR & 26 & 21 & 24 & 18 \\
\hline Hoehn and Yahr score & 3 & 3 & 3 & 3 \\
\hline Stand/walk $7 \mathrm{~m} / \mathrm{sit}(\mathrm{s})$ & 31.7 & 22.2 & 31.9 & 20.7 \\
\hline \multicolumn{5}{|l|}{ Two point test: } \\
\hline Right & 9.1 & 9.2 & 12.5 & 10.3 \\
\hline Left & 10.4 & 8.8 & 13.3 & 9.2 \\
\hline Total dyskinesia score & 0 & 6 & 0 & 5 \\
\hline Contralateral dyskinesia score & 0 & 3 & 0 & 0 \\
\hline \multicolumn{5}{|l|}{ Patient 4 (left pallidotomy): } \\
\hline Motor UPDR & 42 & 29 & 30 & 14 \\
\hline Hoehn and Yahr score & 2.5 & 2.5 & 2.5 & 2.5 \\
\hline Stand/walk $7 \mathrm{~m} /$ sit (s) & 23.5 & 14.7 & 18.4 & 15.2 \\
\hline \multicolumn{5}{|l|}{ Two point test } \\
\hline Right & 12 & 7.6 & 11.1 & 8.2 \\
\hline Left & 12.4 & 8.4 & 12.3 & 8.4 \\
\hline Total dyskinesia score & 0 & 7 & 0 & 5 \\
\hline Contralateral dyskinesia score & 0 & 2 & 0 & 1 \\
\hline
\end{tabular}

Motor UPDR refers to items $18-31$. Point test refers to the time taken to move the index finger 20 times between two points placed $30 \mathrm{~cm}$ apart. Total dyskinesia score equals the sum of five scores (trunk and each limb), each within the range of $0-4$, plus the global dyskinesia score $\left(0-4^{41}\right)$. Contralateral dyskinesia score equals the sum of two scores, each within the range of $0-4$, for the arm and leg contralateral to the lesion.
The aim of the current study was to determine if the preoperative organisation pattern of the reach to grasp movement in Parkinson's disease is altered as a result of posteroventral pallidotomy. For this purpose, intrinsic (size) and extrinsic (distance) properties of the object were manipulated to establish whether both the global parameters, such as movement duration and movement patterning, are affected by surgery. Given the results from previous studies of medicated patients with early stage Parkinson's disease who show normal movement patterning it was predicted that a lesion to the globus pallidus should not disrupt movement patterning. Further, given brain imaging findings ${ }^{27-29}$ of enhanced activity in cortical areas downstream from the globus pallidus interna, and clinical reports of a decrease in bradykinesia after pallidotomy, it was predicted that the reach to grasp movement should be quicker. The results support only this hypothesis but raise some questions about the role of the basal ganglia, in particular, the globus pallidus interna, in movement organisation.

\section{Materials and methods}

PATIENTS

Four patients with Parkinson's disease underwent unilateral pallidotomy: patient 1, a 71 year old woman with Parkinson's disease of 21 years' duration, patient 2, a 54 year old man with Parkinson's disease of 4 years' duration, patient 3, a 75 year old woman with Parkinson's disease of 11 years' duration, and (4) patient 4, a 63 year old man with Parkinson's disease of 12 years' duration. The daily levodopa equivalent dosage ${ }^{30}(100 \mathrm{mg}$ of regular levodopa $=130 \mathrm{mg}$ of controlled release levodopa $=10 \mathrm{mg}$ bromocriptine $=1 \mathrm{mg}$ pergolide) was $610 \mathrm{mg}, 100 \mathrm{mg}, 500 \mathrm{mg}$, and 1245 mg for patients $1,2,3$, and 4 respectively. For three patients $(1,3$, and 4$)$, intervention was suggested because of severe dyskinesia induced by medication, and motor fluctuations. For patient 2, surgery was suggested because of intolerance to levodopa and poor relief of symptomatology with alternative medication. Morning clinical assessments, including CAPIT scores and UPDRS, were performed in the week before, and 1 (patient 1) or 3 (patients 2-4) months postoperatively. These assessments were undertaken in the off state (after 12 hours of withdrawal from parkinsonian medication) and in the best on state, ${ }^{8}$ after the morning medication (patients 1 and 2: $100 \mathrm{mg}$ levodopa/25 mg carbidopa; patients 3 and 4 : $200 \mathrm{mg}$ levodopa/50 mg carbidopa). Table 1 shows the score of each patient for each section of the UPDRS in the off state preoperatively. Table 2 gives the values of the dyskinsia score and some CAPIT scores in off and on states for each patient before and after surgery.

No patients showed visual field defects and all had normal or corrected to normal visual acuity. All patients gave informed consent. The project was approved by both hospital and university ethics committees. 

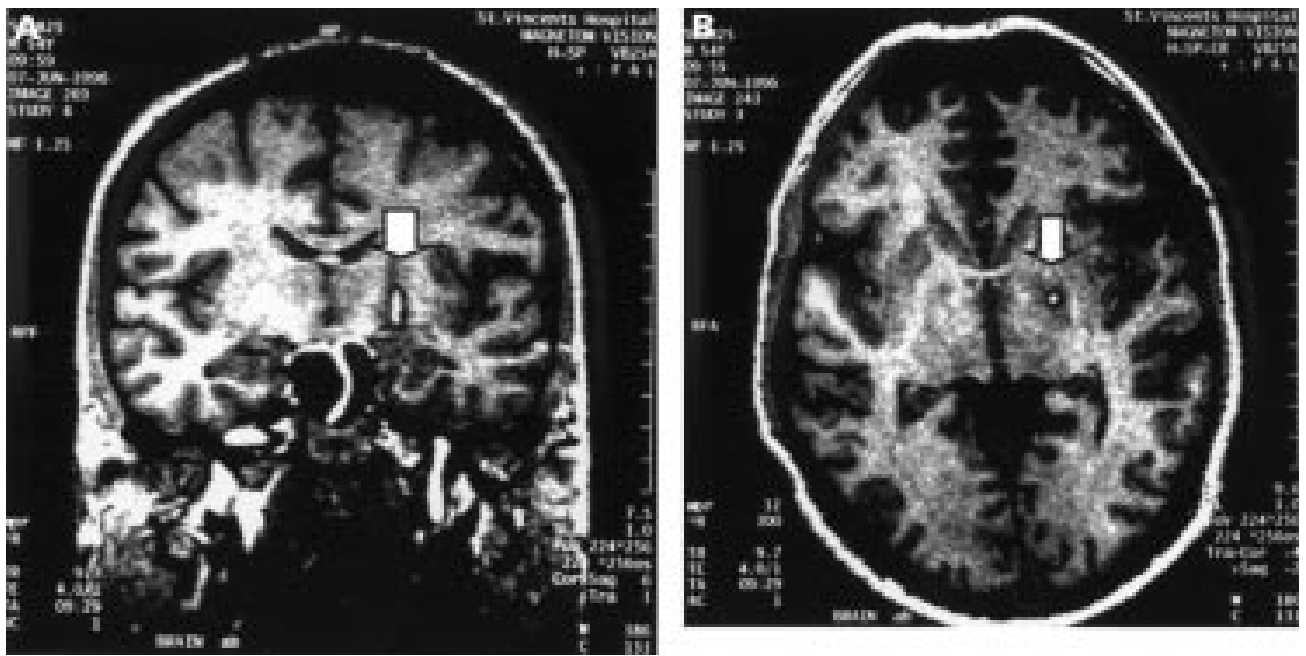

Figure 1 Postoperative axial and coronal MRI of the lesion site to the posteroventral pallidum (patient 2).

SURGICAL PROCEDURE

Surgery took place after withdrawal from Parkinson's disease related medication for at least 12 hours. The lesioning protocol was based on that used by Dogali et al. ${ }^{4}$ With a Cosman-Roberts-Wells (Radionics, Burlington, MA, USA) stereotaxic frame affixed to the patient's skull, a three dimensional volume MRI data set was acquired (Siemens 1.5 Tesla scanner) and images reconstructed parallel to the line joining the anterior and posterior commissures (AC-PC plane) and at right angles to this. The anatomical target (the most posterior and ventral aspect of the globus pallidum interna) was $2-3 \mathrm{~mm}$ anterior to the midcommisural point, 5-6 $\mathrm{mm}$ below the AC-PC line, and $18-21 \mathrm{~mm}$ lateral to the midline. The final preoperative target was selected after inspection of the reformatted axial and coronal images with particular reference to the internal capsule and optic tract.

A $10 \mathrm{~mm}$ frontal burr hole was made $2-3 \mathrm{~cm}$ lateral to the midline positioned so as to make the electrode trajectory $0-20^{\circ}$ to the sagittal plane and $60-80^{\circ}$ to the axial AC-PC plane. Through a $1.5 \mathrm{~mm}$ diameter guide tube extending up to $5 \mathrm{~mm}$ into the cortex, an Ohye semi-microelectrode was introduced into the brain and advanced towards the target. Neuronal recording was started $10 \mathrm{~mm}$ from the target with signals amplified, passed through an $\mathrm{AD}$ converter, and inspected visually and aurally (Viking II Electrodiagnostic System, Nicolet Diagnostic Instruments, Madison WI,

Table 3 Mean (SD) values of selected parameters before surgery in off and on states

\begin{tabular}{|c|c|c|c|c|}
\hline & \multicolumn{4}{|l|}{ Patient } \\
\hline & 1 & 2 & 3 & 4 \\
\hline \multicolumn{5}{|c|}{ Movement initiation time $(\mathrm{ms})$ : } \\
\hline Off & $518(98)$ & $524(63)$ & $411(95)$ & $363(82)$ \\
\hline On & $518(148)$ & $528(74)$ & $401(61)$ & $321(62)$ \\
\hline Difference & - & - & - & - \\
\hline \multicolumn{5}{|c|}{ Movement duration (ms): } \\
\hline Off & $1595(159)$ & $1027(76)$ & $1230(104)$ & $1145(105)$ \\
\hline On & $1217(111)$ & $935(57)$ & $957(60)$ & $998(113)$ \\
\hline Difference & -378 & -92 & -273 & -147 \\
\hline \multicolumn{5}{|c|}{ Time to peak reach velocity $(\%)$ : } \\
\hline Off & $41(8)$ & $38(5)$ & $39(5)$ & $41(5)$ \\
\hline On & $41(6)$ & $41(4)$ & $45(4)$ & $47(5)$ \\
\hline Difference & - & - & +7 & +6 \\
\hline \multicolumn{5}{|c|}{ Reach deceleration time (ms): } \\
\hline Off & $928(121)$ & $637(70)$ & $757(96)$ & $686(91)$ \\
\hline On & $720(100)$ & $560(65)$ & $527(60)$ & $531(86)$ \\
\hline Difference & -208 & -77 & -231 & -155 \\
\hline \multicolumn{5}{|c|}{ Amplitude of maximum grip aperture $(\mathrm{mm})$ : } \\
\hline Off & $82(7)$ & $69(5)$ & $87(6)$ & $72(7)$ \\
\hline On & $97(7)$ & $72(4)$ & $76(5)$ & $60(3)$ \\
\hline Difference & +15 & - & -11 & -12 \\
\hline \multicolumn{5}{|c|}{ Time to peak grip aperture $(\%)$ : } \\
\hline Off & $70(8)$ & $77(9)$ & $70(8)$ & $71(7)$ \\
\hline On & $64(7)$ & $78(6)$ & $69(5)$ & $69(9)$ \\
\hline Difference & -6 & - & - & - \\
\hline \multicolumn{5}{|c|}{ Variability of time to peak deceleration (ms): } \\
\hline Off & 238 & 117 & 95 & 94 \\
\hline On & 137 & 67 & 74 & 174 \\
\hline Difference & -101 & -50 & -22 & +80 \\
\hline \multicolumn{5}{|c|}{ Variability of time to peak grip aperture $(\mathrm{ms})$ : } \\
\hline Off & 125 & 96 & 96 & 79 \\
\hline On & 80 & 56 & 47 & 92 \\
\hline Difference & -45 & -40 & -49 & +13 \\
\hline
\end{tabular}

Differences are given only when significant $t$ test results were obtained $(\mathrm{p}<0.01)$. No tests were performed on the variability values. 

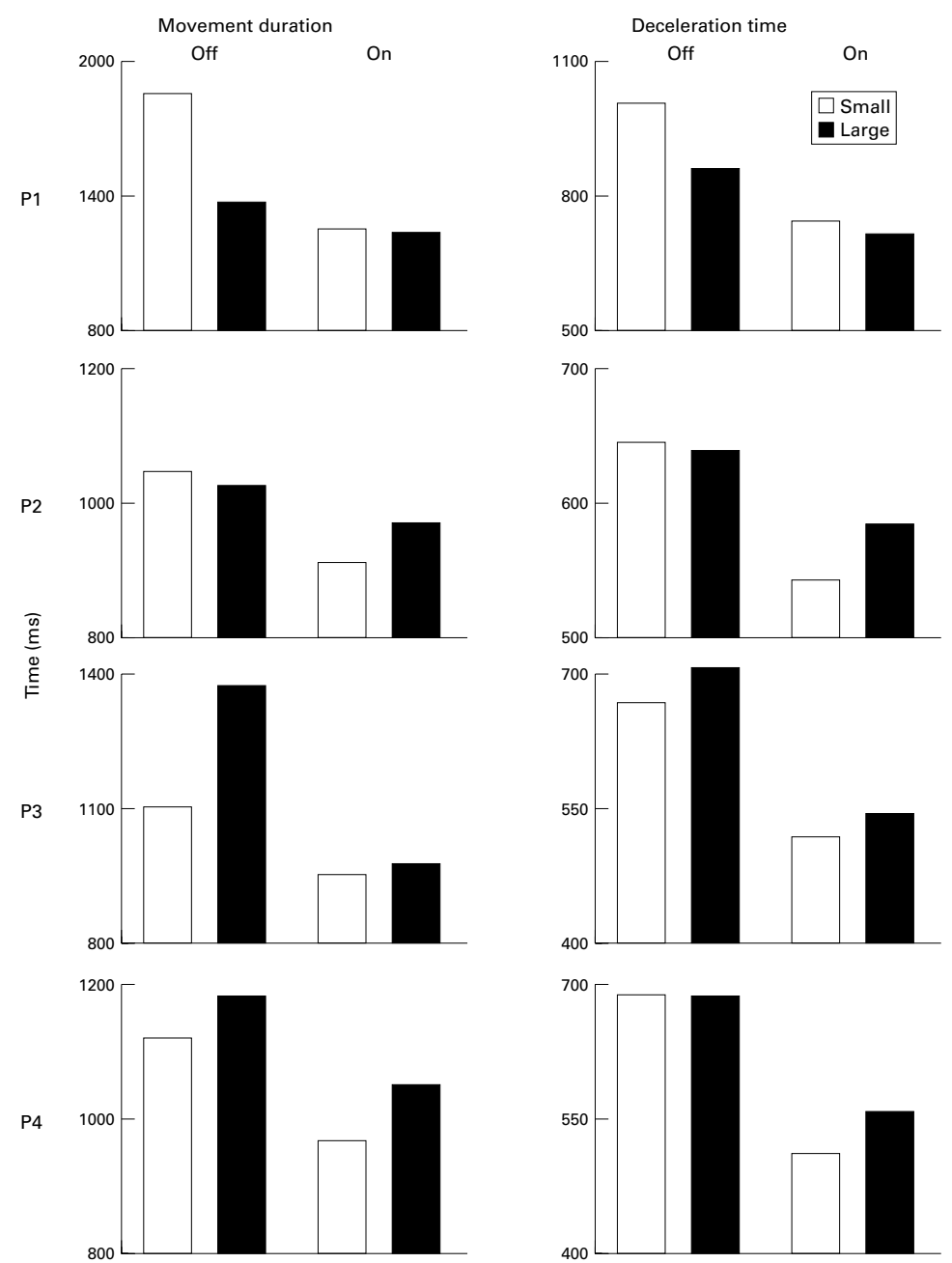

Figure 2 Bar graphs illustrating size patterning preoperatively for each of the patients with Parkinson's disease (patients 1-4) in the on and off states for the parameters of movement duration (left column) and deceleration time (right column).

USA). Based on characteristic neuronal discharge patterns, the ventral and dorsal margins of the globus pallidus were determined.

The semi-microelectrode was then replaced with a lesioning probe ( $1 \mathrm{~mm}$ diameter, $2 \mathrm{~mm}$ exposed tip, Radionics, Burlington, MA, USA) which was lowered under continuous impedance monitoring to $8 \mathrm{~mm}$ above the target. Electrical stimulation through the probe $(0.2$ ms pulse duration, 2, 5, 50, and $100 \mathrm{~Hz}, 0-10$ V) was conducted at 8,4 , and $2 \mathrm{~mm}$ dorsal to the target, at the target, and at $2 \mathrm{~mm}$ ventral to the target. During stimulation, the face and limbs were observed for muscle contraction and patients were asked to report phosphenes, to determine relation to the internal capsule and optic tract respectively. Up to four trajectories (3, 4, 3, and 1 respectively for patients 1-4) were analysed to determine the optimal target. After a test radiofrequency thermocoagulation lesion at $70^{\circ} \mathrm{C}$ for 20 seconds, permanent lesions at $80^{\circ} \mathrm{C}$ for $60 \mathrm{sec}-$ onds were performed at $2 \mathrm{~mm}$ levels from the target to $8 \mathrm{~mm}$ dorsal along the chosen trajectory, to produce a cylindrical lesion. Speech, vision, and motor function were monitored throughout the lesioning process and formal visual fields tested postoperatively. Figure 1 shows an example of the lesion site as confirmed postoperatively (at 1-3 days) by MRI.

KINEMATIC ASSESSMENT PROCEDURE

The kinematic assessment was performed during morning sessions in both off and on states in the week before surgery and 1-2 months after surgery. The day of these assessments differed from that of the clinical assessment. The on state was 1 hour after the following first dose medication for each subject: patient 1 levodopa/benserazide $25 / 6.25 \mathrm{mg}$, levodopa/ benserazide (controlled release) $100 / 25 \mathrm{mg}$, pergolide $0.375 \mathrm{mg}$, amantadine $100 \mathrm{mg}$, selegiline $5 \mathrm{mg}$; patient 2 pergolide $0.5 \mathrm{mg}$, amantadine $100 \mathrm{mg}$; patient 3 levodopa/ carbidopa $100 / 25 \mathrm{mg}$, selegiline $5 \mathrm{mg}$; patient 4 levodopa/carbidopa $125 / 12.5 \mathrm{mg}$, levodopa/ carbidopa (modified release) $200 / 50 \mathrm{mg}$, bromocriptine $10 \mathrm{mg}$.

Movements were recorded with the ELITE three dimensional kinematic analysis system (BITIS| Italy). Greater detail of this system is given in Castiello et al. ${ }^{31}$ The cameras detect infrared reflections of small markers $(0.25 \mathrm{~cm}$ diameter) attached to the following points of the reaching limb: (a) wrist-radial aspect of the distal styloid process of the radius; $(b)$ index finger-radial side of the nail; and (c) thumbulnar side of the nail. Under normal lighting conditions, the participant was seated in front of the table working surface $(1 \times 1 \mathrm{~m})$. Before each trial, the right or the left hand was placed on the table in the mid-sagittal plane $15 \mathrm{~cm}$ from the thorax. In this position the shoulder was flexed $\left(5-10^{\circ}\right)$, the elbow flexed, the forearm semipronated, and the wrist was in $10-15^{\circ}$ of extension. The index finger and thumb were held gently opposed, and the ulnar border of the hand rested on a pressure sensitive starting switch. The target was a perspex cylinder of either small $(0.7 \mathrm{~cm})$ or large $(8 \mathrm{~cm})$ diameter and $8 \mathrm{~cm}$ height placed $20 \mathrm{~cm}$ or $30 \mathrm{~cm}$ directly in front of the starting switch. Each trial began with an acoustic signal representing the "go" command for the patient to reach and grasp the cylinder. No instructions were given as to the speed of movement or its spatial boundaries. For each size/distance combination the participant performed 15 trials with each hand.

The ELIGRASP (B|TISI, 1994) software package was used to give a three dimensional reconstruction of the marker positions and to filter the data. ${ }^{29}{ }^{30}$ The transport component was assessed by analysing the trajectory, velocity, and acceleration profiles of the wrist marker. The manipulation component was assessed by analysing the trajectory of each of the hand markers, and the distance between these two markers. Movement initiation time, so called because no emphasis was placed on a rapid response, was taken from release of the starting switch. The end of the movement was taken as the time when the fingers closed on the object and there was no further change in the distance between the index finger and thumb. The dependent variables were temporal measures of the grasp and reach components (see 
Table 4 Mean (SD) values of selected parameters in the off state before and after surgery

\begin{tabular}{|c|c|c|c|c|}
\hline & \multicolumn{4}{|l|}{ Patient } \\
\hline & 1 & 2 & 3 & 4 \\
\hline \multicolumn{5}{|c|}{ Movement initiation time (ms): } \\
\hline Before & $518(98\}$ & $524(63)$ & $411(95)$ & $363(82)$ \\
\hline After & $517(145)$ & $379(51)$ & $352(54)$ & $369(59)$ \\
\hline Difference & - & -145 & -59 & - \\
\hline \multicolumn{5}{|c|}{ Movement duration (ms): } \\
\hline Before & 1595 (159) & $1027(76)$ & $1230(104)$ & $1145(105)$ \\
\hline After & 1335 (147) & $818(56)$ & $1011(68)$ & $1055(89)$ \\
\hline Difference & -260 & -209 & -219 & -90 \\
\hline \multicolumn{5}{|c|}{ Time to peak reach velocity (\%): } \\
\hline Before & $41(8)$ & $38(5)$ & $39(5)$ & $41(5)$ \\
\hline After & $36(6)$ & $42(4)$ & $39(5)$ & $48(6)$ \\
\hline Difference & -5 & +4 & - & +7 \\
\hline \multicolumn{5}{|c|}{ Reach deceleration time (ms): } \\
\hline Before & $928(121)$ & $637(70)$ & $757(96)$ & $686(91)$ \\
\hline After & $861(114)$ & $479(55)$ & $548(60)$ & $555(83)$ \\
\hline Difference & -67 & -158 & -209 & -131 \\
\hline \multicolumn{5}{|c|}{ Amplitude of maximum grip aperture $(\mathrm{mm})$ : } \\
\hline Before & $82(7)$ & $69(5)$ & $87(6)$ & $72(7)$ \\
\hline After & $51(5)$ & $83(4)$ & $65(4)$ & $105(8)$ \\
\hline Difference & -31 & +14 & -22 & +33 \\
\hline \multicolumn{5}{|c|}{ Time to peak grip aperture (\%): } \\
\hline Before & $70(8)$ & $77(9)$ & $70(8)$ & $71(7)$ \\
\hline After & $68(8)$ & $74(6)$ & $75(7)$ & $71(7)$ \\
\hline Difference & - & - & +5 & - \\
\hline \multicolumn{5}{|c|}{ Variability of time to peak deceleration (ms): } \\
\hline Before & 238 & 117 & 95 & 94 \\
\hline After & 143 & 46 & 85 & 83 \\
\hline Difference & -95 & -71 & -10 & -11 \\
\hline \multicolumn{5}{|c|}{ Variability of time to peak grip aperture $(\mathrm{ms})$ : } \\
\hline Before & 125 & 96 & 96 & 79 \\
\hline After & 110 & 48 & 70 & 70 \\
\hline Difference & -15 & -48 & -26 & -9 \\
\hline
\end{tabular}

Differences are given only when significant $t$ test results were obtained $(\mathrm{p}<0.01)$. No tests were performed on the variability values.

Castiello $e t a l^{\beta 1}$ for details). For each parameter, independent $t$ tests were conducted between (a) the preoperative off and on states to determine the effects of medication preoperatively, and $(b)$ the preoperative and postoperative off states to determine the effects of surgery. Throughout the reporting of the results, differences reflect a $t$ value which was significant at an $\alpha$ level of at least 0.01 .

\section{Results}

CLINICAL TESTS

Table 2 shows a summary of the results of clinical tests conducted before and after unilateral pallidotomy. For all patients the score obtained for the motor component of the UPDRS was highest in the off state preoperatively and lowest in the on state postoperatively, with the mean motor UPDRS motor score in the off state improving by $30 \%$ after surgery. The stand-walk-sit time value was included to give a general idea of the speed of a gross motor action. This time was greater in the off than in the on state and generally decreased postoperatively. The two point test was included to indicate the results for a gross upper limb movement, of relevance to the movement assessed in the current study. However, results for this test were inconsistent. Patients 2 and 4 showed improvement in both limbs after surgery, patient 1 showed slight worsening in the ipsilesional hand, and patient 3 showed a slight bilateral increase in this time. The medication induced dyskinesia of patients 1,3 , and 4 improved postoperatively (mean preoperative score 7.6 , mean postoperative score 6 , representing an average $21 \%$ improvement), particularly in the contralesional limb.
KINEMATIC ASSESSMENT

Preoperative off state $v$ preoperative on state

The patterning of movement before surgery varied greatly across the four patients who participated in this study, and it is because of this heterogeneity that the results for each patient are presented separately.

Reach to grasp kinematics: data collapsed according to object size and target distance

Table 3 shows the mean preoperative values of a selected number of parameters for each subject in the off and on states. Very few showed a common medication effect. One exception was movement duration, which showed a decrease ranging from $92-378 \mathrm{~ms}$ after medication. This decrease in movement duration was supported by significantly increased amplitudes of the peaks of arm reaching acceleration, velocity, and deceleration. Another exception was deceleration time, which showed a clear decrease in absolute terms (range 77-231 ms). By contrast, movement initiation time showed no change with medication, and there were no clear pattern of results for any of the transport or manipulation kinematic parameters. For example, patient 1 showed no change to transport parameters with medication whereas patients 2,3 , and 4 showed later temporal settings of peak velocity, acceleration, and deceleration after medication. Similarly, medication affected the amplitude of maximum grip aperture (the maximum distance between the thumb and index finger markers during the opening/closing cycle) in three of four patients, but the direction of this change varied (for example, it was greater for patient 1 but less for patients 3 and 4 ). Three of the four patients 

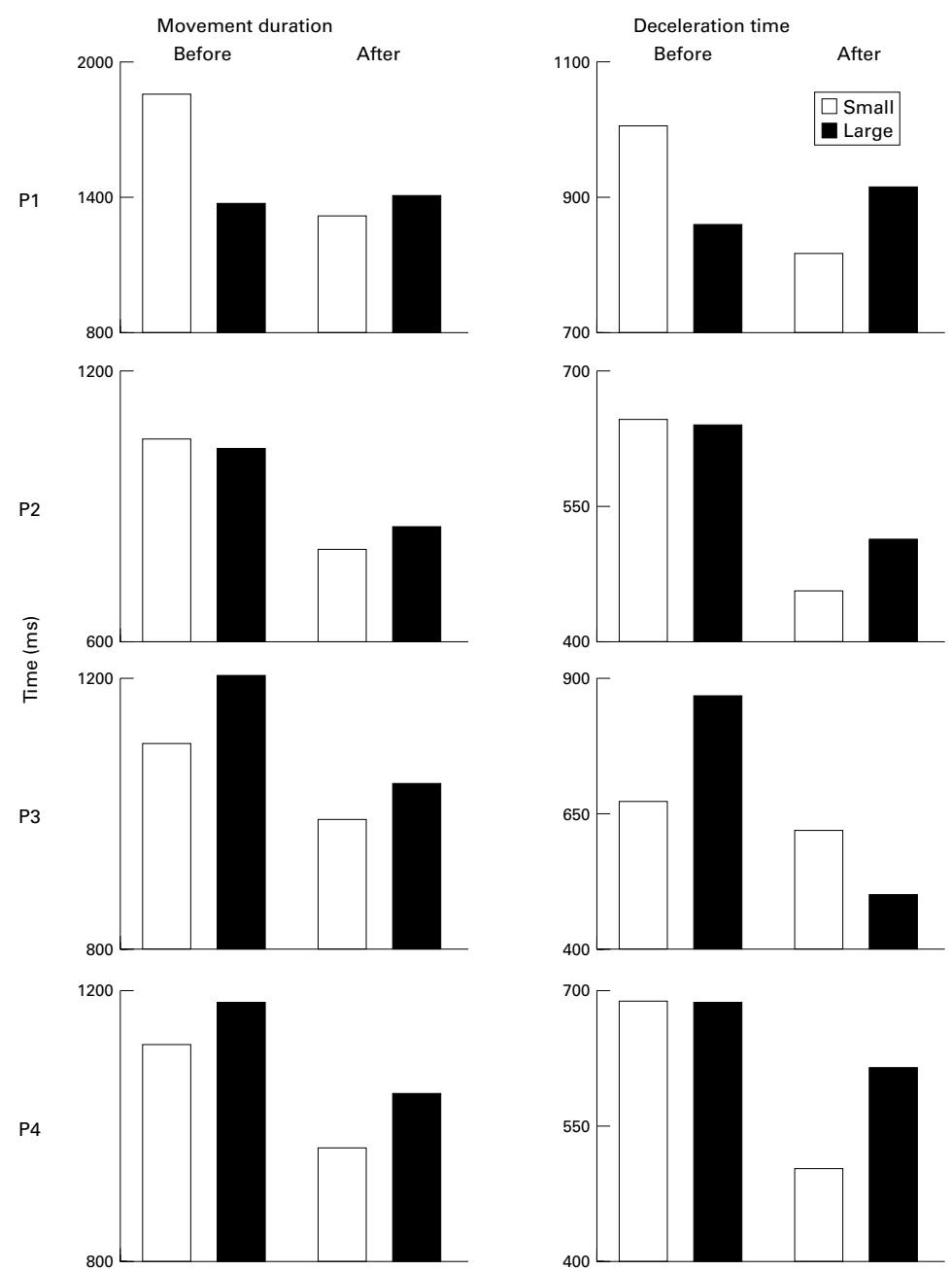

Figure 3 Bar graphs illustrating size patterning preoperatively and postoperatively for each of the patients with Parkinson's disease (patients 1-4) in the off state for the parameters of movement duration (left column) and deceleration time (right column).

also showed an overall decrease in variability with medication.

A comparison of reach to grasp kinematics according to object size (small v large).

Many previous studies have shown that patients with Parkinson's disease in early disease stages and in the on state demonstrate normal movement patterning with regard to object size..$^{20-25}$ However, in the current study it was only the first pallidotomy patient (patient 1) who showed the classic small-large patterning in both the on and off states before surgery. Reflecting the greater accuracy requirements, movement duration for this patient was greater for the small than for the large object, and this was supported by lower amplitudes in the peaks of velocity, acceleration, and deceleration for the small object. Similarly, deceleration time (the time taken for the reaching arm to hone in on the object) was greater for the small than for the large object.

Figure 2 shows that for the parameters of movement duration and deceleration time, medication served to reduce the small/large differences for patient 1 but a normal patterning was preserved. By contrast, medication preoperatively for patient 2 led to an inappro- priate patterning of the transport component with movement duration becoming less for the small than for the large object and deceleration time remaining lower for the small object. Patients 3 and 4 showed inappropriate patterning in both the off and on states preoperatively. In the case of patient 3, for example, both movement duration and deceleration time were less for the small than for the large object.

A common pattern for all four patients was that the manipulation component showed the classic patterning before surgery, with maximum grip aperture occurring earlier for the small than for the large object. This patterning was little influenced by medication.

\section{Comparison of reach to grasp kinematics}

according to reaching distance $(20 \mathrm{~cm} v 30 \mathrm{~cm})$.

All four patients showed normal patterning according to target distance-that is, lower amplitudes of the peaks of velocity, acceleration, and deceleration for the $20 \mathrm{~cm}$ than for the $30 \mathrm{~cm}$ target. The effect of medication on this patterning was minimal. For example, both patient 1 and patient 3 showed decreased differences in variability according to distance for many transport and manipulation component parameters, but no disruption to the normal pattern in the amplitudes of the peaks.

\section{PREOPERATIVE OFF STATE V POSTOPERATIVE OFF} STATE

Reach to grasp kinematics: data collapsed according to object size and target distance. Although all patients showed postoperative changes to various parameters the pattern of this change varied across patients. However, two parameters (movement duration and deceleration time) showed results which were common to all four patients, and several parameters showed a reduction in variability postoperatively. Table 4 shows that the average decrease in movement duration ranged from $90 \mathrm{~ms}$ to $260 \mathrm{~ms}$, similar to the preoperative medication effect. This decrease in movement duration was coupled with the expected increase in the amplitudes of peak reach acceleration, velocity, and deceleration. For all but one patient (4) the reduction to movement duration was bilateral. Patient 1 and patient 3, who had left and right pallidotomy, respectively, showed a greater decrease for the left (patient 1: $329 \mathrm{~ms}$; patient 3: $283 \mathrm{~ms}$ ) than for the right limb (202 ms, $154 \mathrm{~ms}$ ). Patient 2, who had a left pallidotomy, showed a greater decrease in movement duration in the right $(283.5 \mathrm{~ms})$ than in the left limb $(136 \mathrm{~ms})$, and patient 4, who also had a left pallidotomy showed a decrease in the movement duration of the right limb only (by $196 \mathrm{~ms}$ ).

The parameter of deceleration time showed a decrease ranging from $67 \mathrm{~ms}$ to $209 \mathrm{~ms}$ (again similar to the preoperative medication effect). This decrease was bilateral for all four patients. Patients 2, 3, and 4 showed a greater decrease to the right deceleration time. For patient 1 the small decreases $(<75 \mathrm{~ms}$ ) were the same for both limbs.

For many parameters, surgical effects were apparent but differed across patients. Move- 

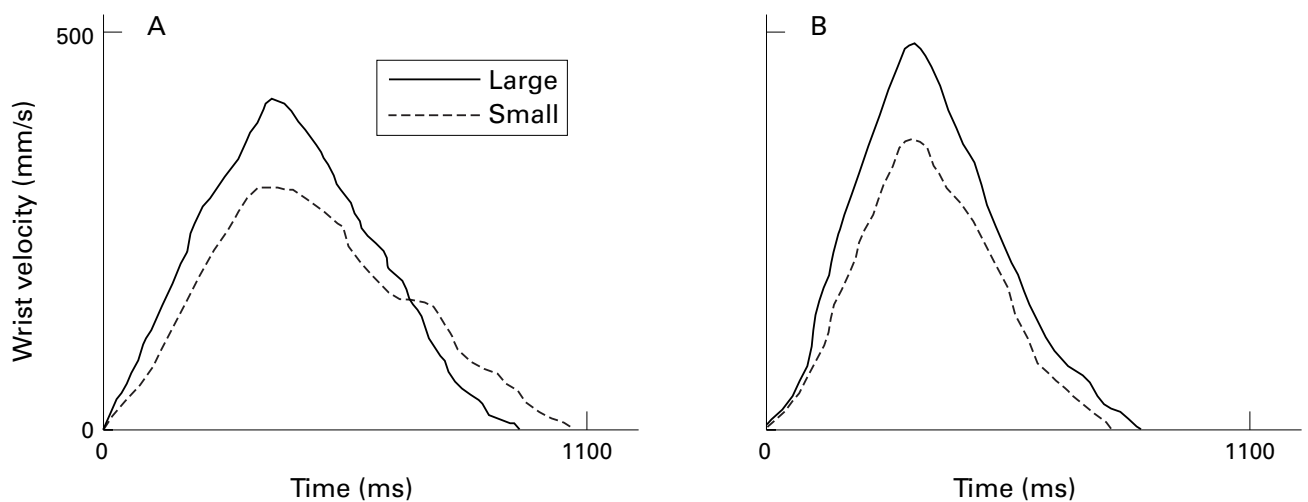

Figure 4 Velocity profiles obtained from reach to grasp movements (patient 2) to the small and large cylinders $(A)$ before and (B) after surgery.

ment initiation time (time from acoustic signal to release of starting switch) was lower for two patients ( 2 and 4 ) postoperatively. Many transport and manipulation component parameters showed no consistent pattern (table 4). For example, peak reach velocity was relatively earlier for patient 1 , later for patient 2 and patient 4 , and showed no change for patient 3 . The amplitude of maximum grip aperture was consistently affected by surgery but the pattern of this change varied across patients.

\section{Comparison of reach to grasp kinematics}

according to object size (small v large)

Surgery clearly affected the kinematics of the transport component according to target object size. Figure 3 illutrates that no patient showed the classic patterning postoperatively. In all four patients postoperatively, movement duration for the small object was less than that for the large object. For patients 1 and 2 this signified a reversal of the normal off preoperative pattern. For patient 4 this signified a worsening of the abnormal patterning and in the case of patient 3 a change to the small/large difference of abnormal patterning. Figure 4 shows an example of velocity profiles obtained from reach-to-grasp movements to the small and large cylinders by patient 2 . Before surgery, movement duration was longer for the small than for the large object. After surgery this pattern was reversed.

The postoperative results for deceleration time were similar with clear changes to the patterning of this parameter for all four patients. Three of the four patients showed an abnormal patterning postoperatively. Again, for patient 1 and patient 2 this signified a reversal of the normal off preoperative patterning, and for patient 4 a worsening of the abnormal patterning. Patient 3 showed the normal patterning of a longer deceleration time for the small than for the large object. This contrasted with the abnormal patterning found preoperatively.

A most unusual result was the relation between movement duration and the amplitude of arm reaching velocity. It is usual to see a higher peak velocity if the movement duration is lower-in other words, the movement is faster. Conversely, a higher movement duration is normally accompanied by a lower peak velocity. However, the postoperative results did not follow this pattern. Even though movement duration was lower for the small than for the large object, the amplitudes of peak reach acceleration and velocity were not greater for the small than for the large object postoperatively (fig 4). Such results indicate that the movement to the small object was completed in a shorter time but with a lower speed than the movement to the large object-that is, the accelerative phase of the movement seemed to be planned appropriately but the decelerative phase, inappropriately, according to size.

Parameters of the manipulation component did not show a clear disruption to the small/large patterning. All four patients showed a postoperative pattern of peak grip aperture being relatively earlier for the small than for the large object. This reflected the pattern found preoperatively. For example, the timing of peak grip aperture for patient 3 in the off state preoperatively was at $64 \%$ of movement duration $(706 \mathrm{~ms})$ for the small object and at $77 \%$ (1049 ms) for the large object. Postoperatively these figures were at averages of $69 \%$ (SD 681 $\mathrm{ms}$ ) and $83 \%$ (SD $870 \mathrm{~ms}$ ), respectively.

\section{A comparison of reach to grasp kinematics} according to reaching distance $(20 \mathrm{~cm} v 30 \mathrm{~cm})$ Patterning according to target distance was not changed as a result of surgery. All four patients continued to show the normal patterning of lower amplitudes of the peaks of velocity, acceleration, and deceleration for the $20 \mathrm{~cm}$ than for the $30 \mathrm{~cm}$ distance. For example, in the case of patient 2, preoperatively the amplitude of the peak of acceleration was 3320 $\mathrm{mm} / \mathrm{s}^{2}$ for the $20 \mathrm{~cm}$ reaching distance and $3723 \mathrm{~mm} / \mathrm{s}^{2}$ for the $30 \mathrm{~cm}$ distance. Postoperatively these values were $5038 \mathrm{~mm} / \mathrm{s}^{2}$ and 6714 $\mathrm{mm} / \mathrm{s}^{2}$ respectively (recalling that movements were of lower duration postoperatively). For the manipulation component, all patients showed the normal patterning of an earlier peak grip aperture for the $20 \mathrm{~cm}$ than for the 30 $\mathrm{cm}$ reach.

\section{Discussion}

The main aim of this study was to determine the effects of unilateral posteroventral pallidotomy on motor actions by assessing kinematically the performance of the everyday action of reaching to grasp an object. The pat- 
terning of this action has been well characterised by a host of previous research studies, with consistent findings that kinematics differ according to intrinsic (for example, size) and extrinsic (for example, distance) characteristics of the object to be grasped. Hence, the interest in this study was in determining the effect of unilateral pallidotomy on these characteristic movement patternings.

At a global level, the clear surgical benefit was to movement speed and duration. Postoperatively, all four patients with Parkinson's disease showed lower movement durations and movements which were of greater velocity. For three patients $(2,3$, and 4$)$, the reduction was primarily due to decreases in the time taken to hone in on the target object (deceleration time). For example, patient 3 showed a reduction in movement duration of $219 \mathrm{~ms}$ postoperatively, with $209 \mathrm{~ms}$ of this being to the deceleration phase. Patient 1 was the only patient to show greater time reductions in the accelerative than in the decelerative phase of movement.

The probable explanation for this enhancement of movement speed is that pallidotomy results in easier excitation of the motor association areas. Using PET, it has been demonstrated that the motor association cortex (supplementary motor area and premotor cortex) shows greater movement related activity after pallidotomy. ${ }^{27-29}$ By removing (or decreasing) inhibitory pallidal influences upon thalamocortical circuitry, the association cortex is thought to be more "ready" or "set" for activation. The reduction in movement duration probably reflects the improved time efficiency of the supplementary motor area and premotor cortex in reaching the threshold for activation.

Counter to the benefits to movement speed are the apparent costs to movement patterning according to size. Postoperatively, no patients showed the normal kinematic patterning of longer movement duration and deceleration time for the small than for the large object. In all four patients, the duration of movement was lower, and the period spent in honing in upon the object was lower, for the small than for the large object. A clear confounding effect in this study is that three patients showed abnormality of the size function before unilateral pallidotomy. In itself, this is contrary to the results of many previous studies of patients with early stage Parkinson's disease in whom no abnormality of movement patterning according to size has been reported. ${ }^{20-25}$ It also increases the difficulty of dissociating pre-existing abnormality from surgical effects. Certainly surgery does not improve or restore the size function. Indeed, the postoperative results indicate that surgery magnifies abnormality in three patients, and detrimentally changes the patterning of the one patient who showed normality preoperatively.

One interpretation of these results is that feedback mechanisms are altered by lesioning the posteroventral pallidum. The main evidence for this is the inappropriate allocation of deceleration time according to object size. This suggests that visual information about object size is not appropriately relayed to motor pathways during the on line performance, particularly during the deceleration phase. At the neurophysiological level, such an interpretation is difficult to defend because the basal ganglia form part of a medial system which operates largely in a feedforward mode (see Goldberg ${ }^{34}$ for review). Lesioning the globus pallidus interna should thus theoretically affect feedforward rather than feedback processes. Yet the accelerative phase of the movement does not show abnormality in the size function postoperatively. Amplitudes of the peaks of arm reaching acceleration and velocity are lower for the small than for the large object. Such a result suggests that the initial part of the movement is patterned correctly, and could be interpreted as reflecting normal feedforward processes.

Many previous studies have shown that patients with Parkinson's disease become more dependent on visual feedback to guide movement. ${ }^{34-37}$ Goldberg $^{34}$ proposed that this could reflect greater reliance upon the responsive, feedback dependent, lateral, premotor system involving the arcuate premotor area and the cerebellum; in other words, the system reverts to alternative and healthier neural channels. With regard to pallidotomy, such a theory would predict no change to this use of feedback mechanisms with lesioning of the globus pallidus interna because the system would still revert to use of the lateral system. By contrast, these results suggest that feedback mechanisms have been disturbed by surgery, and thus point to a role for the basal ganglia in the processing of visual information during the on line performance of a motor action.

There are reasons for exercising caution in adopting the proposal that posteroventral pallidotomy affects feedback mechanisms, or that it promotes greater reliance on feedforward pathways. The first is that only four patients have been investigated, and within this group there was tremendous between patient variability in movement patterning preoperatively. Of note, however, is that the patients of this study showed similar degrees of clinical improvement to those of previous studies. For example, the mean off motor UPDRS score for these four patients demonstrated a 30\% improvement-a result which resembles the $30 \%$ and $24 \%$ improvements reported by Lozano et $a \bar{T}$ and Baron et al, ${ }^{10}$ respectively. Secondly, the effects of surgery are very specific. It is only the patterning of the transport (reach) component that shows the dysfunction. The size function of the manipulation component does not seem to be affected postoperatively, and suggestive of normal patterning, the timing of peak grip aperture is earlier for the small than for the large object. On the one hand this lends support to the argument that transport and manipulation are subserved by separate visuomotor pathways, of which only the transport is targeted by unilateral pallidotomy. An alternative explanation is that the patterning of peak grip aperture timing is under feedforward rather than feedback processing. A third reservation against accepting the feedback theory is that distance patterning of both the transport 
and manipulation components is not affected by surgery. Hence, the peak amplitudes of arm reaching acceleration, velocity, and deceleration are all lower for a $20 \mathrm{~cm}$ than for a $30 \mathrm{~cm}$ distance both preoperatively and postoperatively. Again, however, these parameters may be under feedforward control.

In summary, unilateral posteroventral pallidotomy results in quicker reach to grasp actions but disruption to the kinematic organisation of reach parameters with respect to the intrinsic object characteristics of size. These results raise interesting questions about the function of the globus pallidus interna. Supporting much previous research into the function of the basal ganglia, the current study adds evidence for the notion that these nuclei are not responsible for the selection of specific muscles - there is no obvious disruption to the overt performance of the reach to grasp action as a result of surgery. However, the finding of stimulus bound effects on movement kinematics suggests that the globus pallidus interna plays a part in high level sensorimotor functions which couple particular sensory inputs to appropriate movement patternings. ${ }^{38}{ }^{39}$ This idea is in line with that proposed by Moore ${ }^{40}$ who suggested that the basal ganglia are well placed to play a comparator function comparing motor output to feedback.

The four patients with Parkinson's disease who participated in this study are gratefully acknowledged. Helen Chambers, Mary-Lyn Draper, and Suryani Lim are thanked for their Mary-Lyn Draper, and Suryani Lim are thanked for their Australian National Health and Medical Research Council and Australian Research Council project grants, and by a grant from Australian Research Council project grants,
Merck, Sharp, and Dohme to UC and KB.

1 Laitenen L, Bergenheim A, Hariz M. Leksell's posteroventral pallidotomy in the treatment of Parkinson's disease. $\mathcal{F}$ tral pallidotomy in the tre
Neurosurg 1992;76:53-61.

2 Laitenen L, Bergenheim A, Hariz M. Ventroposterolateral pallidotomy can abolish all parkinsonism symptoms. Stereotact Funct Neurosurg 1992;58:14-21.

3 Svennilson E, Torvik A, Lowe R, et al. Treatment of parkinsonism by stereotaxic thermolesions in the pallidal region. Acta Neurol Scand 1960;35:358-77.

4 Dogali M, Fazzini E, Kolodny E, et al. Stereotaxic ventral pallidotomy for Parkinson's disease. Neurology 1995;45 753-61.

5 Lozano AM, Lang AE, Galvez-Jimenes N, et al. GPi pallidotomy improves motor function in Parkinson's disease. Lancet 1995;346:1383-6.

6 Johansson F, Malm J, Nordh E, et al. Usefulness of pallidotomy in advanced Parkinson's disease. 7 Neurol Neurosurg Psychiatry 1997;62:125-32.

7 Fahn S, Elton RI members of the UPDRS Developmen Committee. Unified Parkinson's disease rating scale. In: Fahn S, Marsden CD, Calne DB, et al, eds. Recent developFahn S, Marsden CD, Calne DB, et al, eds. Recent developments in Parkinson's disease. Vol 2. Florham Park,

8 Langston JW, Widner H, Goetz CG, et al. Core assessment program for intracerebral transplantations (CAPIT). Mov program for intracer

9 Dogali M, Sterio D, Fazzini E, et al. Effects of posteroventral pallidotomy. Adv Neurol 1996;69:585-90.

10 Baron MS, Vitek JL, Bakay RAE, et al. Treatment of advanced Parkinson's disease by posterior GP pallidotomy: 1-year results of a pilot study. Ann Neurol 1996;40:355-66.

11 Obeso JA, Linazasoro G, Rothwell JC, et al. Assessing the effects of pallidotomy in Parkinson's disease. Lancet 1996; 347:1490.

12 Kelly PJ. Pallidotomy in Parkinson's disease. Neurosurgery 1995;36:1154-7.
13 Lozano AM. Pallidotomy: where are we? Parkinsonism Related Disorders 1996;2:53-4.

14 Olanow CW. GPi pallidotomy-have we made a dent in Parkinson's disease? Ann Neurol 1996;40:341-3.

15 Obeso JA, Guridi J, DeLong M. Surgery for Parkinson's disease. F Neurol Neurosurg Psychiatry 1997;62:2-8.

16 Bennett KMB, Peppard RF, O'Sullivan JD, et al. Visuospatial attentional functioning and movement kinematics preand post-pallidotomy. In: Castiello U, ed. Mechanisms of perception for the control of action. Monash University, Victoria, Australia; 1996:81-90.

17 Simuni T, Shils J, Stecker M, et al. Effects of pallidotomy on Parkinson's patients; comparisons on a computer trajectory Parkinson's patients; comparisons on a computer
paradigm [abstract]. Mov Disord 1996;11:158.

18 Fookson O, Berkinblit M, Hening W, et al. Pallidotomy improves the accuracy and trajectory of 3-dimensional pointing movements in Parkinson's disease: a case study [abstract]. Mov Disord 1996;11:186.

19 Jeannerod $M$. The timing of natural prehension movements. f Mot Behav 1984;16:235-54.

20 Bennett KMB, Adler CH, Stelmach GE, et al. A kinematic study of the reach to grasp movement in a subject with hemiParkinson's disease. Neuropsychologia 1993;31:70916.

21 Castiello U, Bennett KMB. The bilateral reach to grasp movement of Parkinson's disease subjects. Brain 1997;120: 593-604.

22 Castiello U, Bennett KMB, Adler CH, et al. Perturbation of the grasp component of a prehension movement in a subject with hemiParkinson's disease. Neuropsychologia 1993; 31:717-23.

23 Castiello U, Stelmach GE, Lieberman AN. Temporal dissociation of the prehension pattern in Parkinson's disease. Neuropsychologia 1993;31:395-402.

24 Castiello U, Bennett KMB, Scarpa M. The reach to grasp movement of Parkinson's disease subjects. In: Bennett $\mathrm{KMB}$, Castiello $\mathrm{U}$, eds. Insights into the reach to grasp movement. Amsterdam: North-Holland, 1994:215-37.

25 Castiello U, Bennett KMB. Parkinson's disease: reorganisation of the reach to grasp movement in response to perturbation of the distal motor patterning. Neuropsychologia 1994;32:1367-82.

26 Scarpa M, Castiello U. Perturbation of a prehension movement in Parkinson's disease. Mov Disord 1994;9:415-25.

27 Eidelberg D, Moeller JR, Ishikawa T, et al. Regional metabolic correlates of surgical outcome following unilateral pallidotomy for Parkinson's disease. Ann Neurol 1996; 39:450-9.

28 Grafton ST, Waters C, Sutton J, et al. Pallidotomy increases activity of motor association cortex in Parkinson's disease: a positron emission tomographic study. Ann Neurol 1995;37:776-83.

29 Ceballos-Baumann AO, Obeso JA, Vitek JL, et al. Restoration of thalamocortical activity under posteroventral pallidotomy in Parkinson's disease. Lancet 1994;344:814.

30 Sinemet CR. For Parkinson's disease. Med Lett Drugs Ther 1991;33:92-3.

31 Castiello U, Bennett KMB. Reach to grasp: the response to a simultaneous perturbation of object position and size. Exp Brain Res 1998;120:31-40.

32 D'Amico M, Ferrigno, G. Technique for the evaluation of derivatives from noisy biomechanical displacement data using a model-based bandwidth-selection procedure. Med Biol Eng Comput 1990;28:407-15.

33 D'Amico M, Ferrigno, G. Comparison between the more recent techniques for smoothing and derivative assessment in biomechanics. Med Biol Eng Comput 1992;30:193-204.

34 Goldberg G. Supplementary motor area structure and function: review and hypotheses. Behav Brain Sci 1985;8: $567-616$

35 Cooke JD, Brown JD, Brooks VB. Increased dependence on visual information for movement control in patients with Parkinson's disease. Can f Neurol Sci 1978;5:413-5.

36 Day BL, Dick JPR, Marsden CD. Patients with Parkinson's disease can employ a predictive motor strategy. $\mathcal{F}$ Neurol Neurosurg Psychiatry 1984;47:1299-306.

37 Flash T, Inzelberg R, Schechtman E, et al. Kinematic analysis of upper limb trajectories in Parkinson's disease. Exp Neurol 1992;118:215-26.

38 Lidsky TI, Manetto C, Schneider JS. A consideration of sensory factors involved in motor functions of the basal ganglia. Brain Res Rev 1985;9:133-46.

39 Kimura $M$. The role of the primate putamen neurons in the association of sensory stimuli with movement. Neurosci Res 1986;3:436-43

40 Moore AP. Impaired sensorimotor integration in parkinsonsm and dyskinesia: a role for corollary discharges? 7 Neurol Neurosurg Psychiatry 1987;50:544-52.

41 Goetz CG, Stebbins GT, Shale HM, et al. Utility of an objective dyskinesia rating scale for Parkinson's disease: inter and intrarater reliability assessment. Mov Disord 1994;9:390-4. 